\title{
Function and Characterization of an Alanine Dehydrogenase Homolog From Nocardia seriolae
}

\author{
Guoquan Chen ${ }^{1,2 \dagger}$, Ziyang Tan ${ }^{1}$, Yansheng Liu ${ }^{1,2}$, Tingting Weng ${ }^{1,2}$, Liqun Xia ${ }^{1,2 \star \dagger}$ and \\ Yishan $\mathrm{Lu}^{1,2 *}$ \\ ' Guangdong Provincial Key Laboratory of Pathogenic Biology and Epidemiology for Aquatic Economic Animals, Fisheries \\ College of Guangdong Ocean University, Zhanjiang, China, ${ }^{2}$ Guangdong Provincial Engineering Research Center for Aquatic \\ Animal Health Assessment, Shenzhen Public Service Platform for Evaluation of Marine Economic Animal Seedings, Shenzhen \\ Institute of Guangdong Ocean University, Shenzhen, China
}

OPEN ACCESS

Edited by:

Lixing Huang,

Jimei University, China

Reviewed by:

Jie Li,

Chinese Academy of Fishery Sciences (CAFS), China

Weiwei Zhang,

Ningbo University, China

${ }^{*}$ Correspondence:

Liqun Xia

xialq@gdou.edu.cn

Yishan Lu

fishdis@163.com

tORCID:

Guoquan Chen orcid.org/0000-0002-0847-0408

Liqun Xia

orcid.org/0000-0001-6244-8060

Specialty section

This article was submitted to Veterinary Infectious Diseases,

a section of the journal

Frontiers in Veterinary Science

Received: 26 October 2021 Accepted: 22 November 2021 Published: 12 January 2022

Citation:

Chen G, Tan Z, Liu Y, Weng T, Xia L and $L u Y$ (2022) Function and Characterization of an Alanine Dehydrogenase Homolog From

Nocardia seriolae.

Front. Vet. Sci. 8:801990 doi: 10.3389/fvets.2021.801990
Fish nocardiosis is a chronic, systemic, granulomatous disease in aquaculture. Nocardia seriolae has been reported to be one of the main pathogenic bacteria of fish nocardiosis. There are few studies on the associated virulence factors and pathogenesis of $\mathrm{N}$. seriolae. Alanine dehydrogenase (ALD), which may be a secreted protein, was discovered by analysis using bioinformatics methods throughout the whole genomic sequence of $N$. seriolae. Nevertheless, the roles of ALD and its homologs in the pathogenesis of $N$. seriolae are not demonstrated. In this study, the function of $N$. seriolae ALD (NSALD) was preliminarily investigated by gene cloning, host cell subcellular localization, secreted protein identification, and cell apoptosis detection. Identification of the extracellular products of $N$. seriolae via mass spectrometry (MS) analysis revealed that NsALD is a secreted protein. In addition, subcellular localization of NsALD-GFP recombinant protein in fathead minnow (FHM) cells showed that the strong green fluorescence co-localized with the mitochondria. Moreover, apoptosis assays demonstrated that the overexpression of NSALD induces apoptosis in FHM cells. This study may lay the foundation for further exploration of the function of NSALD and facilitate further understanding of the pathogenic mechanism and the associated virulence factors of N. seriolae.

Keywords: Nocardia seriolae, alanine dehydrogenase, secreted protein, mitochondrial localization, apoptosis

\section{INTRODUCTION}

Fish nocardiosis, a chronic systemic granulomatous disease, has great influence on both marine and freshwater aquaculture industry $(1,2)$. Nocardia salmonicida, Nocardia asteroids, and Nocardia seriolae have been isolated from diseased fish and confirmed as the pathogenic bacteria of fish nocardiosis (3). Remarkably, $N$. seriolae has been most frequently reported as the main pathogen in the last 30 years. Nocardia seriolae can infect the immunodeficient fish via wounds, the gills, and feeds (4). The symptoms of diseased fish include skin ulceration and serious sarcoidosis caused by a mass of white nodules in the gills, spleen, liver, head kidney, and trunk kidney (5). According to reports, N. seriolae was able to infect about 42 kinds of marine and freshwater fish, such as blotched snakehead (Channa maculata), amberjack (Seriola dumerili), yellowtail (Seriola quinqueradiata), snubnose pompano (Trachinotus blochii), golden pompano (Trachinotus ovatus), 
largemouth bass (Micropterus salmoides), large yellow croaker (Larimichthys crocea), and red drum (Sciaenops ocellatus) (3, 68).

The virulence factors and pathogenic mechanisms of $N$. seriolae-host interaction are not fully studied. It was reported that the virulence of Nocardia species is related to their resistivity to oxidative damage of macrophages, inhibition of a combination of phagosomes and lysosomes, alteration of lysosomal enzymes in phagocytes, and neutralization of the acidification of the phagosome, and some extracellular products seemed to participate in the above processes in Nocardia asteroides (9-12). Our previous studies also indicated that the MTSP3141, GluNS, NsHLP, PLC, SOD, robl/LC7, PTP, and NlpC/P60 of N. seriolae are able to lead to apoptosis in fish cells and are the potential virulence factors of $N$. seriolae. MTSP3141 and GluNS were also confirmed to be secreted proteins and mitochondrial targeting secretory proteins (MTSPs) (7, 13-15). The disease mechanisms of Nocardia sp. have been demonstrated to be varied and complicated and needed further clarification.

In this study, an alanine dehydrogenase (ALD) homolog of $N$. seriolae $(N s A L D)$ was amplified by gene cloning. Then, the secreted proteins were identified and the subcellular localization of NsALD and its involvement in disease mechanisms were evaluated. This study will put forward further clarification of the role of NsALD during infection and facilitate new insights into the molecular pathogenicity of $N$. seriolae.

\section{MATERIALS AND METHODS}

\section{Bacterial Strains, Cell Line, and Plasmids}

Nocardia seriolae ZJ0503 strain (16), fathead minnow (FHM) cells (17), plasmid PEGFP-N1, plasmid PCDNA3.1-His A, and Escherichia coli $\mathrm{DH} 5 \alpha$ were conserved in our laboratory. Nocardia seriolae ZJ0503 isolated from diseased fish was cultured in optimal medium to clone the $A L D$ gene. Escherichia coli DH5 $\alpha$ transformed by plasmid DNA was cultured to extract large numbers of endotoxin-free plasmids. FHM cells (17) transformed by endotoxin-free plasmids were cultured in Leibovitz's L15 medium with $10 \%$ fetal bovine serum for apoptosis assays. The endotoxin-free plasmids pEGFP-N1 and pCDNA3.1-His A were used for subcellular localization and overexpression, respectively.

\section{Gene Cloning and Sequence Bioinformatics Analysis of NsALD}

Nocardia seriolae ZJ0503 was collected after 5 days of cultivation in optimal medium to extract genomic DNA using TIANamp Bacteria DNA Kit (Tiangen, Beijing, China). NsALD was cloned via PCR with two pairs of primers designed using Primer Premier 5.0 software, pEGFP-ALD-F/R and pcDNAALD-F/R, shown in Table 1. Based on the whole-genome sequence data of the $N$. seriolae strain ZJ0503 (accession no. NZ_JNCT01000022), BLAST sequence analysis was carried out through NCBI (http://www.ncbi.nlm.nih.gov/BLAST/). The amino acid sequence for NsALD was predicted and the physicochemical property predicted using ExPASy software (http://www.expasy.org/). Multiple sequence alignment was done with DNAMAN software. A biological evolutionary tree was
TABLE 1 | Primers used for gene cloning in this study.

\begin{tabular}{lll}
\hline Primer name & Sequence $\left(\mathbf{5}^{\prime} \mathbf{-}^{\prime} \mathbf{\prime}\right)$ & $\begin{array}{l}\text { Restriction } \\
\text { enzyme }\end{array}$ \\
\hline pEGFP-ALD-F & GGAATTCATGCTGTTCGATAGCGGCATC & EcoR I \\
pEGFP-ALD-R & CCGACGTCGACTGGGAGGCGATGCGGGTCAC & Sall \\
pCDNA-ALD-F & GGAATCATGCTGTTCGATAGCGGCATC & EcoR I \\
pCDNA-ALD-R & CCGCTCGAGGGAGGCGATGCGGGTCACC & Xho I \\
\hline
\end{tabular}

TABLE 2 | Primers used for qRT-PCR in this study.

\begin{tabular}{lll}
\hline Primer name & Sequence $\left(\mathbf{5}^{\prime} \mathbf{3}^{\prime} \mathbf{)}\right.$ & Gene name \\
\hline Bad-F & TGATCCTTCAGGCGGAGATCTCGC & Bad \\
Bad-R & CAGACTCTTGTGACTCCAAAGGAA & \\
Bid-F & CTGCTCTCCTTCCTTCTTGAGC & Bid \\
Bid-R & GATCAACTCAGCAGCCATATCCCT & \\
Bax-F & TGGCACTGTTCACCTCG & Bax \\
Bax-R & ATCCTCCTTGCTGTCTGATC & \\
Bcl-2-F & TGGGACTGTTGCCTCG & Bcl-2 \\
Bcl-2-R & TCTGCCGCTGCATCTTT & \\
$\beta$-actin-F & ACAATCAATACGGCTGCCATGG & $\beta$-actin \\
$\beta$-actin-R & TTGGCATACAGGTCCTTACTTACGT & \\
\hline
\end{tabular}

constructed using the evolutionary analysis software MEGA 6.0. LocTree 3 (https://rostlab.org/services/loctree3/) and PSORT II Prediction (https://psort.hgc.jp/form2.html) were utilized to predict the subcellular localization and signal peptides.

\section{Plasmid Construction}

Using the two pairs of primers listed in Table 1, the gene NsALD was cloned into pcDNA3.1/His $\mathrm{A}$ and pEGFP-N1 vectors to explore the molecular function and subcellular localization of NsALD in vitro. The constructed recombinant plasmids were subsequently confirmed by sequencing.

PCR was performed with TaKaRa Ex Taq ${ }^{\circledR}$ polymerase using the following PCR program: pre-denaturation at $95^{\circ} \mathrm{C}$ for $5 \mathrm{~min}$, 34 cycles at $95^{\circ} \mathrm{C}$ for $30 \mathrm{~s}, 55^{\circ} \mathrm{C}$ for $30 \mathrm{~s}, 72^{\circ} \mathrm{C}$ for $1 \mathrm{~min}$, and a final extension at $72^{\circ} \mathrm{C}$ for $3 \mathrm{~min}$. The PCR products of NsALD were electrophoresed on $1 \%$ agarose gel and purified using EasyPure PCR Purification Kit (TransGen, Beijing, China). The purified PCR products were digested by the corresponding restriction enzymes, ligated into the eukaryotic vectors pEGFP$\mathrm{N} 1$ and pcDNA3.1/His A, and then transformed into competent E. coli $\mathrm{DH} 5 \alpha$ cells. The different constructs were confirmed by corresponding restriction enzyme digestion (Table 1) and DNA sequencing by Guangzhou Sangon Biological Engineering \& Technology and Service Co. Ltd. Finally, the recombinant plasmids named as pEGFP-ALD and pcDNA-ALD.

\section{Preparation and Identification of Extracellular Products}

After culturing in optimal medium for 3-5 days, single colonies were selected to prepare bacterial suspension. Sterilized 
A

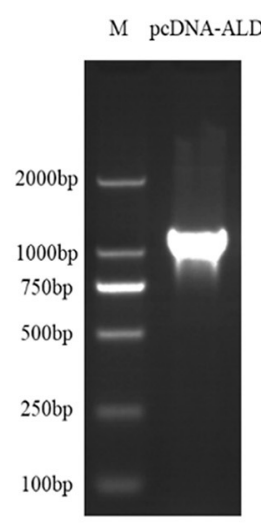

M PEGFP-ALD

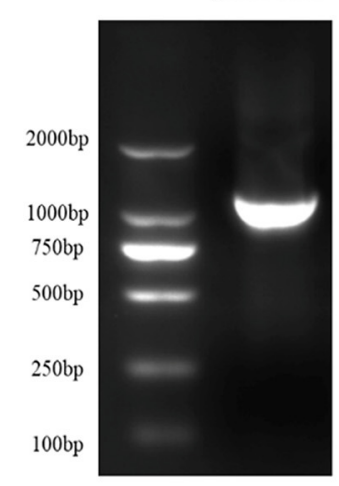

B

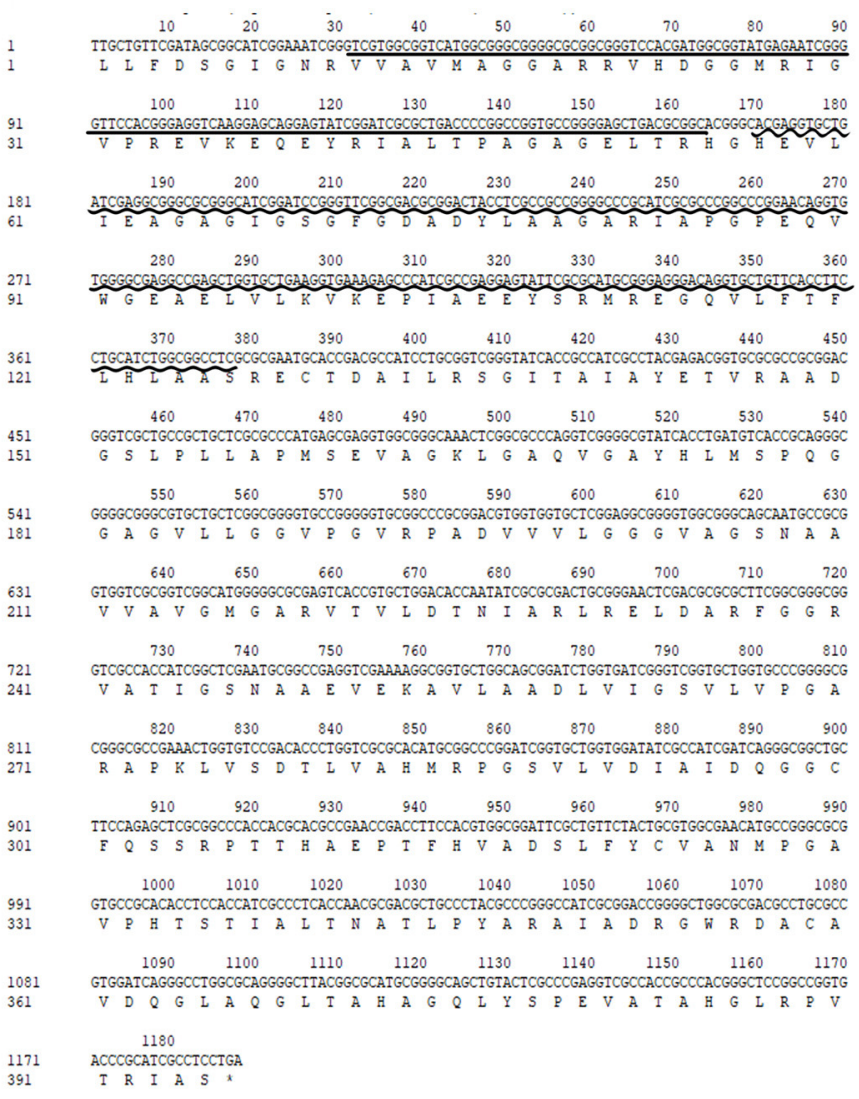

C

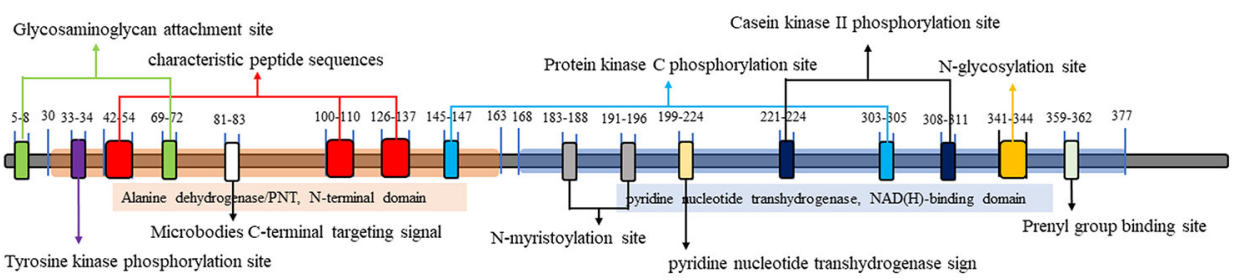

FIGURE 1 | Sequence and structure analysis of the alanine dehydrogenase of Nocardia seriolae (NSALD). (A) NsALD was amplified via PCR using the primers pEGFP-ALD-F/R and pcDNA-ALD-F/R. (B) NsALD nucleic acid sequence and its derived amino acid sequence. The straight line indicates the ALD/PNT, N-terminal domain and the wavy line shows the ALD NAD-binding and catalytic domains. (C) Schematic representation of the prosites of protein NsALD. The NsALD protein comprised two complexity domains (30-163 and 168-377) and nine kinds of functional sites. 
A
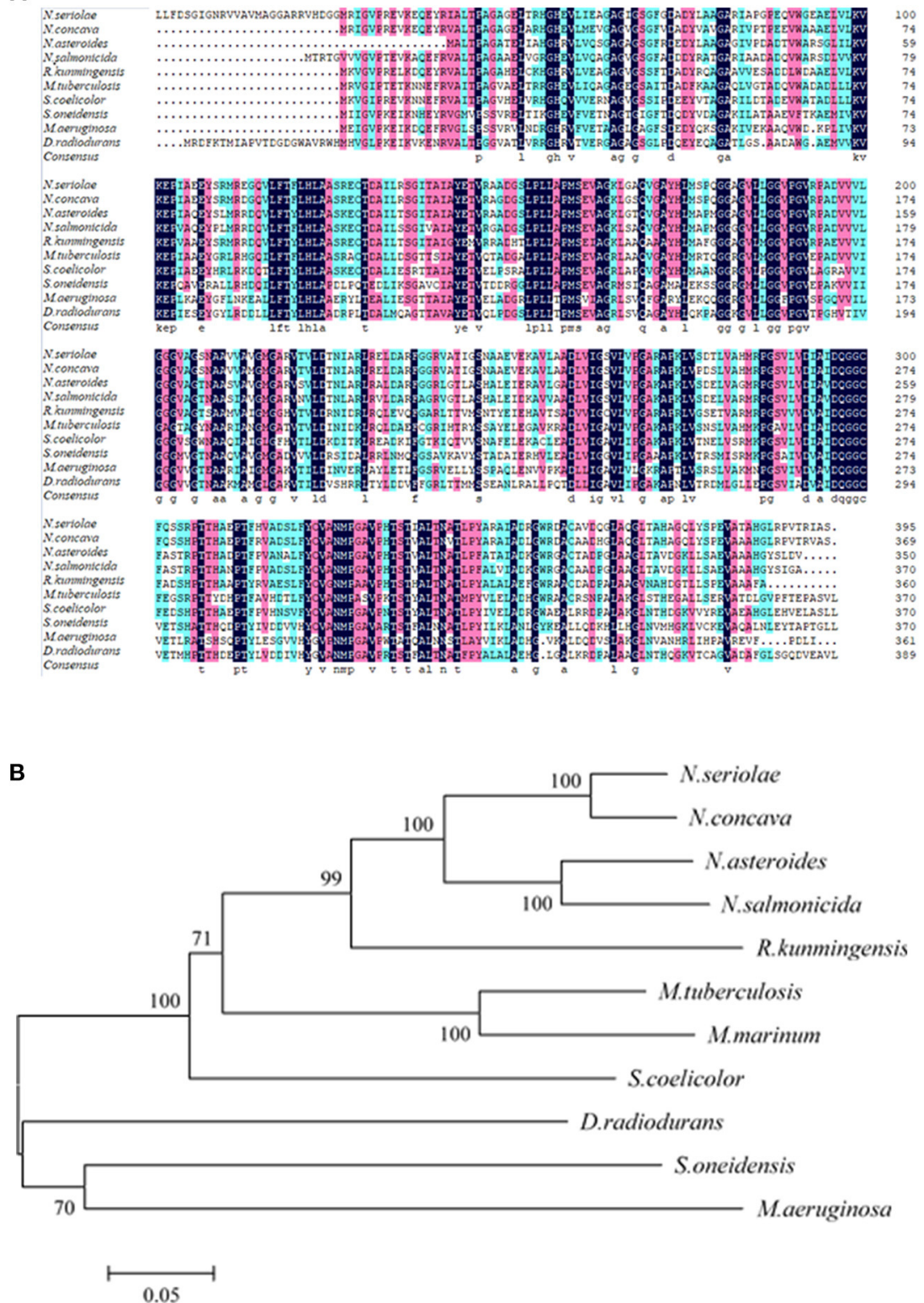

c

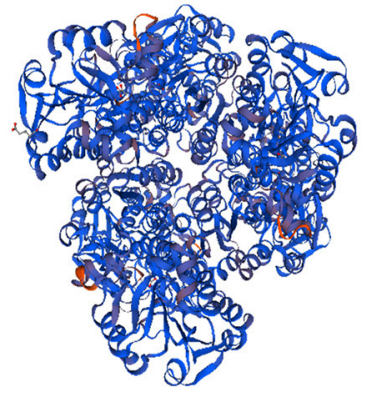

N. seriolae

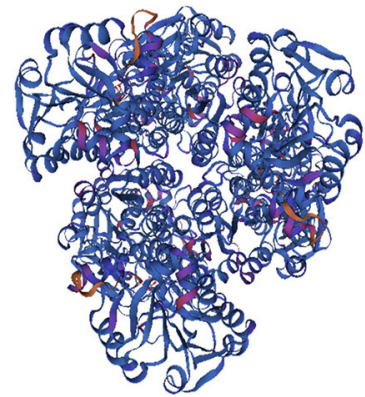

N. concava

FIGURE 2 | Multiple sequence alignment, construction of a phylogenetic tree, and the three-dimensional structure of Nocardia seriolae alanine dehydrogenase (NsALD) protein. (A) Multiple alignment of the deduced amino acid sequences of NsALD protein among different species. Black shaded backgrounds indicate $100 \%$ 
FIGURE 2 | homology, pink shaded backgrounds indicate > 75\% homology, and blue shaded backgrounds indicate $>50 \%$ homology. (B) Construction of a phylogenetic tree among $N$. seriolae and other species with protein NSALD homologous sequences. The protein sequences were aligned using DNAMAN software, and the non-rooted neighbor joining tree was generated by the MEGA 5.0 program. Number at branch points indicates bootstrap support. (C) Structure analysis of NsALD from N. seriolae. The predicted three-dimensional structure of NsALD protein from N. seriolae (left) and Nocardia concava (right). The diagrams were generated using SWISS-MODEL online. GenBank accession numbers are shown as follows: N. seriolae ZJ0503, WP_033090406.1; N. concava, WP_040806553.1: Nocardia asteroides, SFM85941.1; Nocardia salmonicida, WP_062992005.1; Rhodococcus kunmingensis, WP_068269631.1; Mycobacterium tuberculosis H37Rv, NP_217296.1; Mycobacterium marinum, WP_020728350.1; Streptomyces coelicolor A3(2), NP_626044.1; Shewanella oneidensis MR-1, YP_007001355.1; Microcystis aeruginosa, WP_012268249.1; and Deinococcus radiodurans R1, NP_295618.1.

cellophane was laid flat on the modified medium plate as close to the medium as possible. Of the bacterial suspension, $100 \mu \mathrm{l}$ was spread evenly on the medium covered with cellophane and incubated at $28^{\circ} \mathrm{C}$ for 3-5 days. The cellophane with colonies from the medium was removed with tweezers and the colonies on the cellophane rinsed into a sterile beaker with sterile phosphatebuffered saline (PBS). The collected liquid was transferred into a 50 -ml centrifuge tube at $8,000 \times g$ at $4^{\circ} \mathrm{C}$ for $20 \mathrm{~min}$. After centrifugation, the supernatant was sterilized using a $0.22-\mu \mathrm{m}$ microporous membrane and then transferred into a dialysis bag. Dialysis was performed at $4^{\circ} \mathrm{C}$ for $10-16 \mathrm{~h}$ in ultrapure water, during which the dialysate was changed 3 times. After dialysis, the samples were transferred into a $50-\mathrm{ml}$ centrifuge tube and the liquid was frozen into solid at $-80^{\circ} \mathrm{C}$ before freeze drying in a vacuum freeze dryer. The freeze-dried extracellular product samples were sent to a related biological company for protein shotgun LC-MS identification.

\section{Cell Transfection and Subcellular Localization}

Plasmids pEGFP-ALD and pEGFP-N1 were extracted in large quantities via Endo-Free Plasmid Mini Kit I D6948 (Omega BioTek, Norcross, GA, USA). Cell transfection was carried out to explore the subcellular localization of $N s A L D$ in host cells using the Lipofectamine 3000 reagent. At $48 \mathrm{~h}$ post-transfection (hpt), pEGFP-ALD-transfected FHM cells and pEGFP-N1-transfected cells were both stained with $4^{\prime}, 6$-diamidino-2-phenylindole (DAPI) and Mito Tracker Red according to the protocols. After staining and washing with sterilized PBS, the cells were examined under a fluorescence microscope.

\section{Detection of Cell Apoptosis Induced by Overexpression of NsALD Protein}

To determine whether the overexpression of NsALD induces apoptosis in fish cells, transient transfection of FHM cells was carried out using pcDNA-ALD as an experimental group or plasmid pcDNA3.1/His A as a control group. The overexpression of NsALD in FHM cells was verified by Western blot. Then, FHM cells were dyed with DAPI at $48 \mathrm{hpt}$ and observed using a fluorescence microscope. Furthermore, the caspase-3 activity and mitochondrial membrane potential $\left(\Delta \Psi_{\mathrm{m}}\right)$ of the transfected cells were detected, respectively (18) with a caspase-3 colorimetric assay kit K106-25 (BioVision, Milpitas, CA, USA) and a mitochondrial membrane potential assay kit with JC-1 (Beyotime, Shanghai, China).

\section{Quantitative Analysis of the mRNA Expression of Apoptosis-Related Genes}

Quantitative PCR primers (Table 2) for the genes Bcl-2, Bax, Bid, and Bad of FHM cells were designed using Primer Premier 5.0 software. FHM cells transfected with pcDNA-ALD and pcDNA3.1/His A were collected at 0, 24, 48, and 72 hpt. Total RNA was extracted using the TransZol Up Plus RNA Kit (TransGen) and cDNA was synthesized with the EasyScript ${ }^{\circledR}$ One-Step gDNA Removal and cDNA Synthesis SuperMix (TransGen) for real-time quantitative PCR (RT-qPCR) with PerfectStart ${ }^{\circledR}$ Green qPCR SuperMix (TransGen). The effect of transfection on the expressions of apoptosis-related genes was calculated by LightCycler 96 software for RT-qPCR. Each test was performed with the $\beta$-actin gene as the internal control, and there were 3 replicates. The PCR reaction volume was $10 \mu \mathrm{l}, 1 \mu \mathrm{l}$ for each primer $(10 \mu \mathrm{M}), 1 \mu \mathrm{l}$ for cDNA, $2 \mu \mathrm{l}$ for PCR grade water, and $5 \mu$ for Green qPCR SuperMix. The PCR procedures for the four apoptosis-related genes and $\beta$-actin were as follows: $95^{\circ} \mathrm{C}$ for $2 \mathrm{~min}, 40$ cycles of $95^{\circ} \mathrm{C}$ for $75 \mathrm{~s}$, and $55^{\circ} \mathrm{C}$ for $1 \mathrm{~min}$.

\section{Data Analysis}

The comparative $2^{(-\Delta \Delta C t)}$ method was used to calculate the relative expression levels of the four apoptosis-related genes. The data obtained were analyzed with one-way ANOVA using GraphPad Prism 8.0 software. The data were edited and plotted into histograms, and the expression of each apoptosis-related gene at $0 \mathrm{hpt}$ was calculated as 1 . Asterisks show statistical significance, as follows: $p>0.05$, not significant; ${ }^{*} p<0.05$, significant; and ${ }^{* *} p<0.01$, extremely significant.

\section{RESULTS}

\section{Sequence Characterization and Bioinformatics Analysis of NsALD}

NsALD was cloned via PCR using pEGFP-ALD-F/R and $\mathrm{pcDNA}-\mathrm{ALD}-\mathrm{F} / \mathrm{R}$ (Figure 1A). Sequencing analysis showed that the total length of the open reading frame (ORF) of $N s A L D$ was successfully acquired, which encoded an alanine dehydrogenase homolog. The results of sequencing analysis revealed that the ORF of the NsALD gene (GenBank: 61148960) was 1,188 bp encoding 395 amino acid sequences, with alanine dehydrogenase/PNT, N-terminal domain, and alanine dehydrogenase NAD-binding and catalytic domains, which belong to the NADB-Rossmann superfamily (Figure 1B). For subcellular localization, NsALD was predicted to locate in the mitochondria with $81 \%$ expected accuracy using LocTree 3 , while 
it was predicted to locate in the cytoplasm with $94.1 \%$ expected accuracy using PSORT II Prediction. No signal peptide or transit peptide was found. Schematic representation of the prosites demonstrated the protein NsALD to comprise two complexity domains (30-163 and 168-377) and nine kinds of functional sites (Figure 1C).

BLAST protein analysis showed that the NsALD amino acid sequence had high homology with other ALD sequences in actinomycetes, with 92.41, 79.05, 76.15, 68.29, 66.67, 62.60, 52.30, 50.68 , and $53.66 \%$ identity to the ALDs of Nocardia concava, $N$. asteroides, N. salmonicida, Rhodococcus kunmingensis, Mycobacterium tuberculosis H37Rv, Streptomyces coelicolor A3(2), Shewanella oneidensis MR-1, Microcystis aeruginosa, and Deinococcus radiodurans R1, respectively (Figure 2A). A biological evolutionary tree was constructed with the amino acid sequences of 12 bacterial ALDs, which shows that NsALD had a rather high homology among the Nocardia species (Figure 2B). Structure analysis showed a high similarity between the ALDs from N. seriolae and Nocardia concave (Figure 2C).

\section{Detection of Subcellular Localization of NsALD in Transfected Cells}

To explore the subcellular localization of the NsALD protein, the recombinant plasmid pEGFP-ALD was transfected into FHM cells, which was tested by green fluorescence signals. The nucleus was shown as blue fluorescence and the mitochondria was displayed as red fluorescence. In pEGFP-N1-transfected cells, the green fluorescence was dispersed in the whole cell of FHM cells, the mitochondria were distributed in the perinuclear cytoplasm, and the nucleus margin was smooth and had no apoptosis feature (Figure 3, right). Differently, in pEGFP-ALDtransfected cells, nuclear shrinkage appeared, the mitochondria aggregated near the nucleus, and a strong green fluorescence of ALD-GFP exhibited an aggregated distribution near the nucleus and overlapped with the location of the mitochondria, which indicated that the protein NsALD was co-localized with the mitochondria (Figure 3, left).

\section{Secreted Protein Identification to NsALD}

The secreted proteins of $N$. seriolae were acquired and identified with shotgun mass spectrometry. The results revealed that three characteristic peptide sequences of NsALD (IALTPAGAGELTR, SGITAIAYETVR, and VKEPIAEEYSR) were tested with a confidence $\geq 99 \%$, which demonstrated that NsALD is a secreted protein of Nocardia seriolae.

\section{Apoptosis Detection Induced by the Overexpression of NsALD}

To explore whether the NsALD protein has an influence on the apoptosis of fish cells, the control plasmid and the recombinant plasmid pcDNA-ALD were transfected into FHM cells. At $48 \mathrm{hpt}$, several apoptotic bodies can be observed in the transfected FHM cells (Figure 4A), and Western blot showed that the NsALD protein was expressed (Figure 4B). Apoptotic bodies were counted to calculate the apoptosis rate. The result revealed that there were highly significant differences between the experimental group and the control group (Figure 4C). As

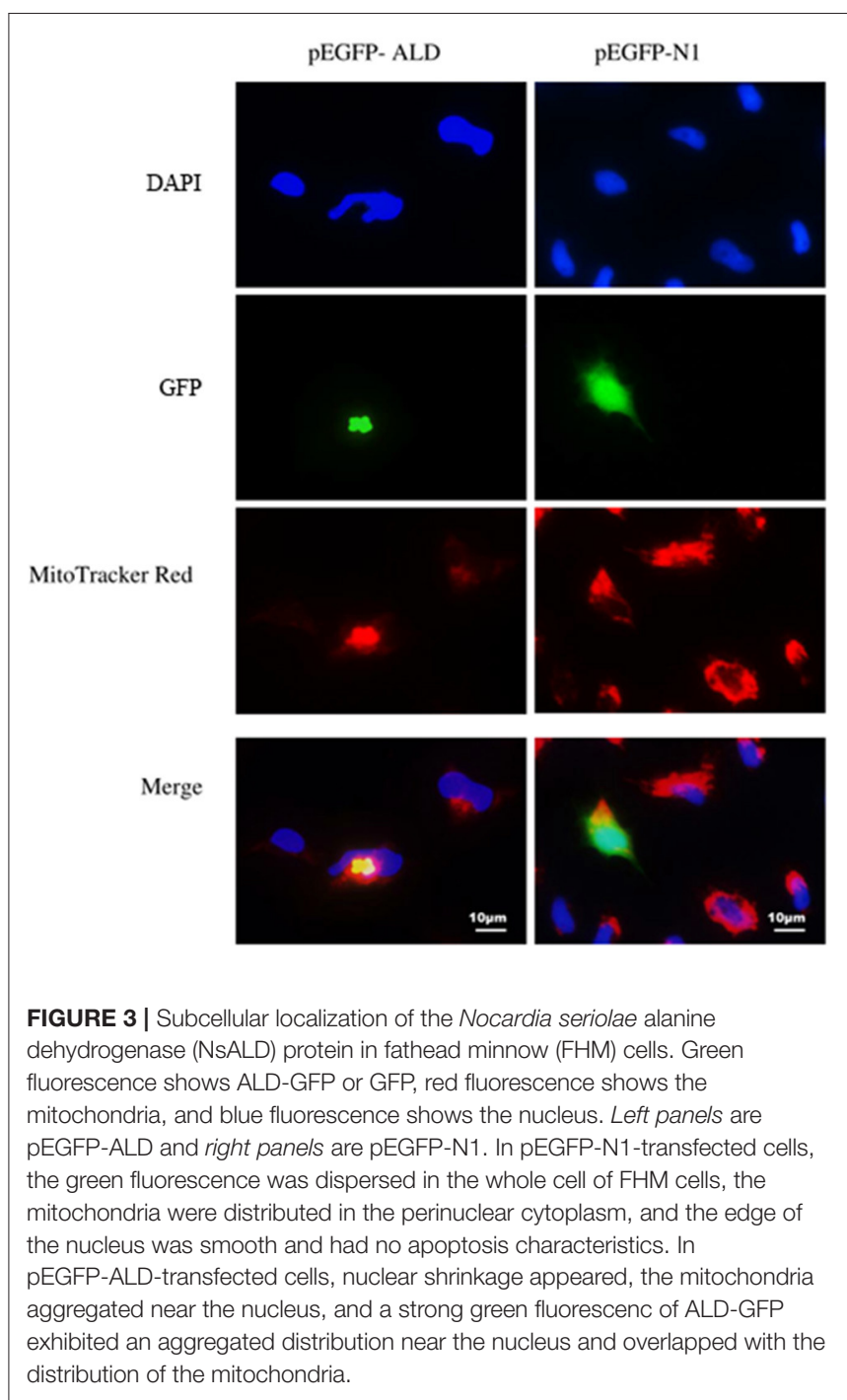

exhibited in Figure 4D, detection of caspase- 3 activity revealed that the maximum value appeared at $48 \mathrm{hpt}$, which was a 1.63 fold increase compared to that of the control group. Furthermore, the $\Delta \Psi_{\mathrm{m}}$ values of NsALD-overexpressing cells compared with those of control cells were reduced remarkably. The $\Delta \Psi_{\mathrm{m}}$ values of NsALD-overexpressing cells decreased to minimum values at $48 \mathrm{hpt}$, a decrease of 0.43 -fold compared to the control group (Figure 4E).

\section{Quantitative Detection of Apoptosis-Related Genes in FHM Cells}

The expression level of each apoptosis-related gene at 0 hpt was considered as the control group. The expressions of Bad and Bax genes increased significantly at 24-72 hpt, with peak values of 10.5 -fold at $72 \mathrm{hpt}$ and 2.9 -fold at $48 \mathrm{hpt}$, respectively. The Bid gene increased significantly at $48-72 \mathrm{hpt}$, reaching a peak value of 2.5 -fold at $72 \mathrm{hpt}$. Interestingly, the expression of $\mathrm{Bcl}-2$, which is an anti-apoptotic gene, showed no change during $0-48 \mathrm{hpt}$, while it increased significantly at $72 \mathrm{hpt}$. Since a high $\mathrm{Bax} / \mathrm{Bcl}-2$ 

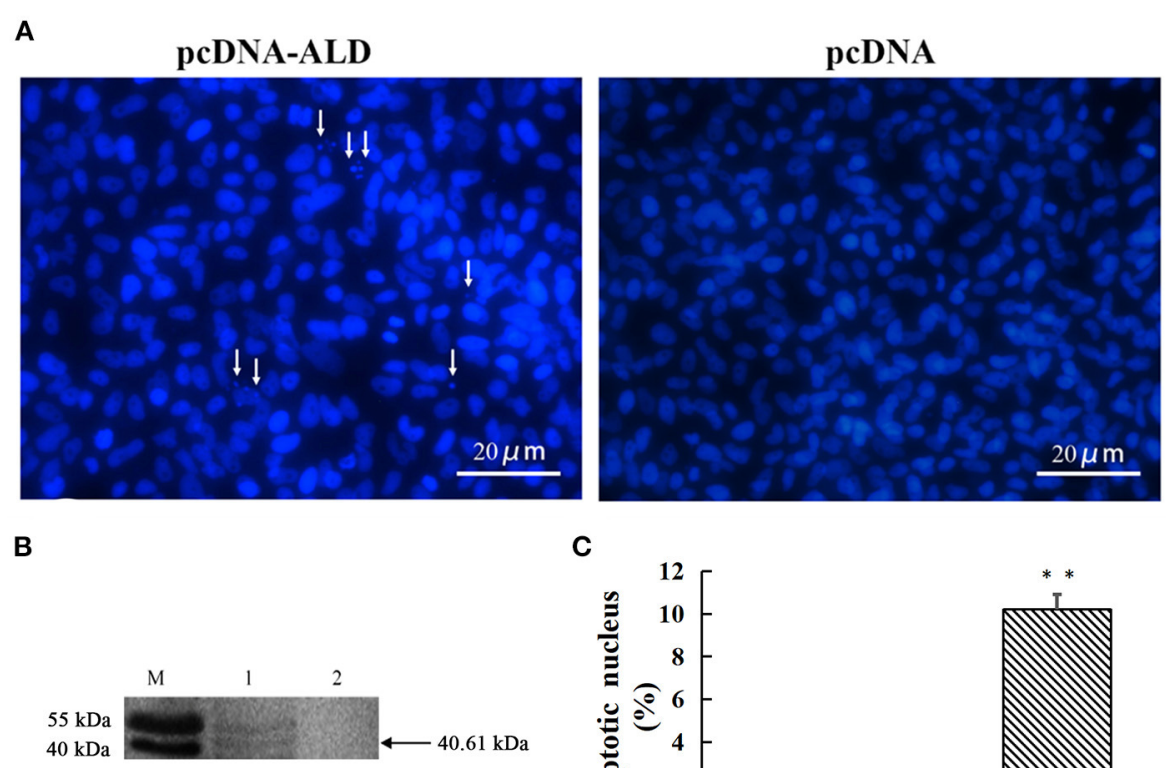

C

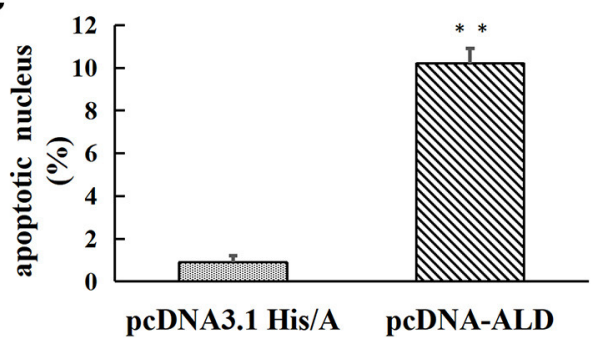

D

$\mathbf{E}$
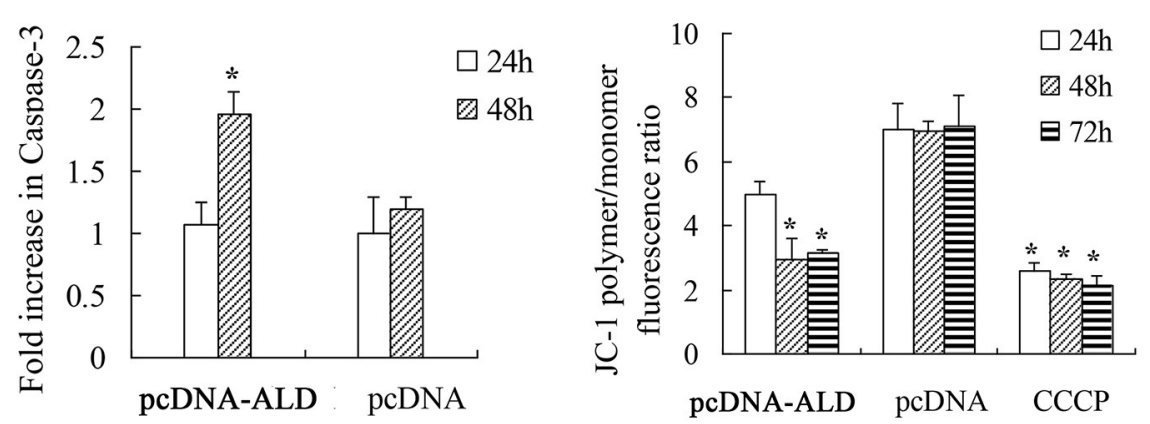

FIGURE 4 | Apoptosis characteristics in pcDNA-ALD-transfected fathead minnow (FHM) cells. (A) Overexpression of protein NsALD (Nocardia seriolae alanine dehydrogenase) in FHM cells. White arrows indicate apoptotic bodies. (B) Confirmation of NsALD expression in FHM cells by Western blot. M, marker. 1, NsALD; 2 , pcDNA. (C) Percentage of apoptotic body in plasmid pcDNA-ALD- or pcDNA-transfected cells. Error bars indicate SD (**p $<0.01)$. (D) Fold increase in caspase-3 after plasmid pcDNA-ALD or pcDNA transfection of FHM cells. FHM cells transfected with plasmids were collected at indicated points after transfection and the levels of cleaved caspase-3 measured. Error bars indicate SD ( $\left.{ }^{*} p<0.05\right)$. (E) Detection of $\Delta \Psi_{m}$ values. FHM cells transfected with plasmid pcDNA-ALD or pcDNA were collected at indicated time points after transfection and the values accessed using JC-1. Untransfected cells treated with carbonyl cyanide $m$-chlorophenylhydrazone (CCCP) were positive controls. Data are expressed as the JC-1 polymer/monomer fluorescence ratio. Error bars indicate SD $\left({ }^{*} p<0.05\right)$.

ratio is related to cell apoptosis, the $B a x / B c l-2$ ratio was calculated according to their expressions. The $B a x / B c l-2$ ratio increased significantly at $24-48 \mathrm{hpt}$, with a peak value of 10.5 -fold at 48 hpt, and then it decreased to 0.5 times that of the control group at $72 \mathrm{hpt}$ (Figure 5). This meant that, although the expression of $\mathrm{Bcl}-2$ increased significantly, the $\mathrm{Bax} / \mathrm{Bcl}-2$ ratio is still low at 72 hpt.

\section{DISCUSSION}

Alanine dehydrogenase (ALD) is a microbial enzyme that catalyzes the reversible oxidation of the deamination of alanine to pyruvate. ALD interconversion between alanine and pyruvate is the core of microbial metabolism (19). ALD was firstly found in Bacillus subtilis by Wiame and Pierard (20). The pathogenicity of bacteria is closely associated with its pathogenic factors. However, only a few studies have been conducted on the virulence factor of $N$. seriolae. Interestingly, in some bacteria, ALD was found to be an antigen-secreting protein in vitro and was regarded as a virulence factor of pathogenic bacteria. For instance, ALD was found in $M$. tuberculosis culture filtrate and identified as one of its main antigens (21). Transcriptional induction of the $A L D$ gene has been observed upon infection of leopard frogs with Mycobacterium marinum, which indicated that ALD may be crucial during M. marinum infection (22). So far, information about the regulation of ALD and its possible role in pathogenesis 
Bid

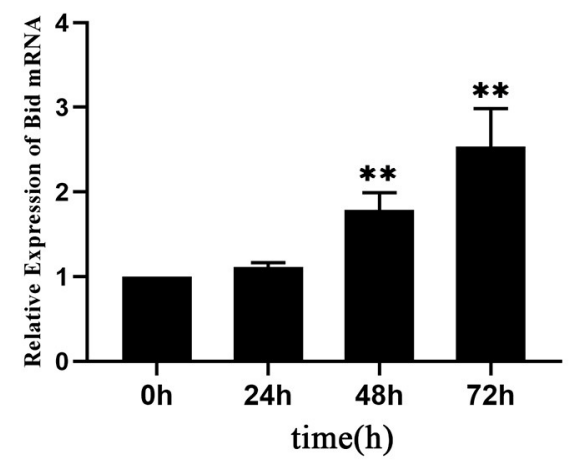

Bax

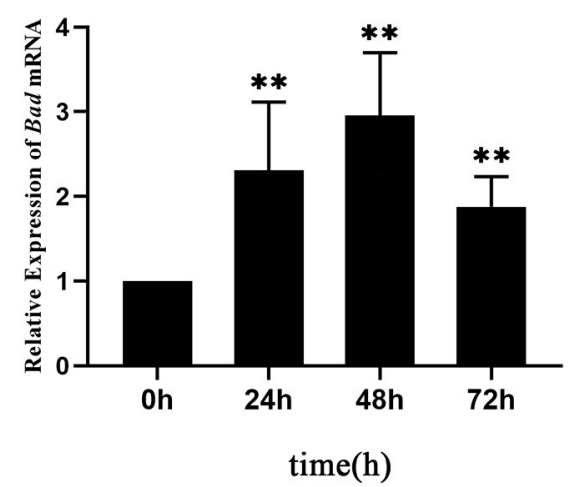

Bad

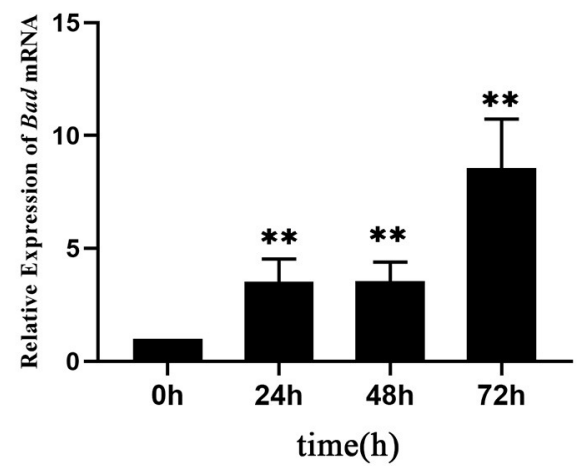

Bcl-2

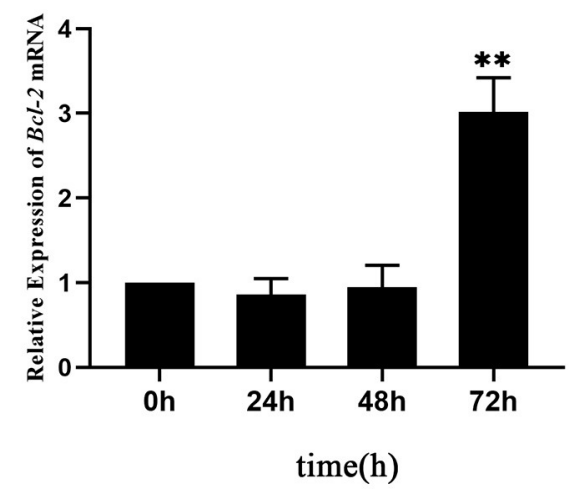

$\mathrm{Bax} / \mathrm{Bcl}-2$ ratio

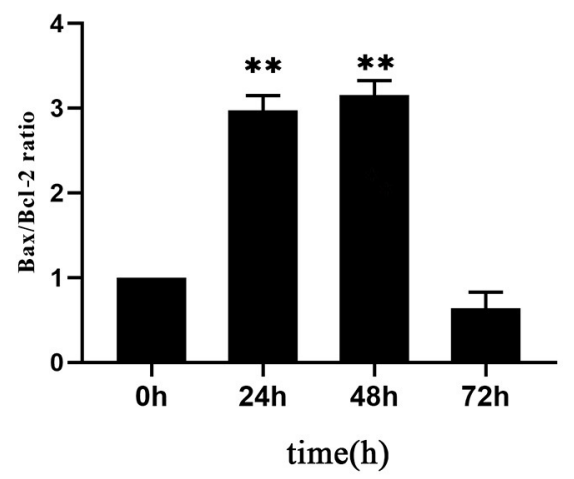

FIGURE 5 | Real-time quantitative PCR (RT-qPCR) analysis of the expressions of apoptosis-related genes in Nocardia seriolae alanine dehydrogenase (NsALD)-overexpressing fathead minnow (FHM) cells. Significant differences are indicated by ${ }^{\star \star} p<0.01$.

is limited (18), and no study related to the ALD of N. seriolae (NsALD) has been reported.

In this study, NsALD was obtained and identified as a secreted protein without a signal peptide. It has been reported that the ALD of $M$. tuberculosis is a predominant antigen in its culture filtrate, which means that ALD is a secreted protein of $M$. tuberculosis; the ALD of M. tuberculosis also lacks a signal peptide (23). NsALD was found to co-localize with the mitochondria in this research. Some proteins targeting the mitochondria contain a transit peptide, while no transit peptide was found in the NsALD protein by bioinformatic prediction. Mitochondrial targeting of bacterial proteins has been reported. Some proteins from parasitic microorganisms such as rickettsial postulated peptidase (RPP) have been discovered to be similar to the protein subunit of mitochondrial processing peptidase (MPP) (24). Additionally, some bacterial virulence factors contain Nterminal mitochondrial targeting signals $(25,26)$. The host cells are invaded by certain proteins of Gram-negative bacteria through the type 3 secretion system (TTSS) and are co-positioned with the mitochondria of host cells (25-27). Helicobacter pylori 
VacA-targeted mitochondria was associated with the formation of anion-selective channels in mitochondrial membranes (28). Further research on the mitochondria-targeting mechanism of NsALD is needed.

The mitochondria is an important organelle with various important functions, such as participating in innate and adaptive immunity and acting as the signal center in cell apoptosis (29), and is also the key target of bacterial invasion (30). Most of the identified bacterial proteins targeting the mitochondria are involved in cell apoptosis, which is consistent with the core effect of the mitochondria in apoptosis regulation (31). For example, the hlyA gene of enteropathogenic E. coli (EPEC) encodes hemolysin, which is targeted to the mitochondria and triggers the apoptosis of host cells via mitochondrial pathways (25). In the process of infection with macrophages, the secreted SopA protein of Salmonella enterica locates in the mitochondria to activate the caspase- 1 independent pathway via the TTSS and leads to macrophage death (32). Cytotoxin VacA related to gastric epithelial lesions is a virulence factor of pathogenic $H$. pylori (33). It was found that $\mathrm{p} 34$, a subunit of VacA, specifically locates in host mitochondria and induces the release of host cytochrome C, which activates caspase- 3 and leads to cell death (34). In this study, typical apoptosis characteristics, such as apoptotic bodies, $\Delta \Psi_{\mathrm{m}}$ values dropping, and caspase- 3 activation, were examined in NsALD-expressed cells, which indicated that NsALD induces apoptosis in host cells.

In our previous work, the secreted virulence factors of $N$. seriolae have also been identified to induce cell apoptosis. The phospholipase $\mathrm{C}$ of $N$. seriolae was a secreted protein and induced apoptosis in FHM cells (15). The MTSP3141 of $N$. seriolae was proven to be a secreted protein, co-located with the mitochondria, and had a 30 -amino acid transit peptide at the $\mathrm{N}$-terminal (35). Glutamyl endopeptidase was a secreted protein without a transit peptide, localized in the mitochondria, and was also a virulence factor of $N$. seriolae that induced apoptosis (36). In addition, the superoxide dismutase (SOD) and histone-like DNA-binding protein (HLP) of N. seriolae was also identified as secreted proteins that lead to apoptosis in host cells $(7,13)$. Taken together, secreted protein-induced apoptosis is associated with $N$. seriolae infection.

According to relevant articles, the main apoptosis pathways in fish are the extrinsic/death receptor pathway (37) and the intrinsic/Bcl-2-regulated/mitochondrial pathway (38). In addition, like mammals, fish have similar functions of conserved members of the Bcl-2 family and inherent apoptosis pathways. Among them, the Bax/Bak-like proteins (Bax, Bak, Bok, Bcl$\mathrm{xs}$, and $\mathrm{Mtd}$ ) and the BH3-only proteins (Bad, Bid, Bik, Bim, Bmf, and Noxa) are pro-apoptotic molecules (39). According to the results of the RT-qPCR of the four apoptosis-related genes, three pro-apoptotic genes (Bax, Bad, and Bid) and the Bax/Bcl2 ratio were significantly activated at $24-48 \mathrm{hpt}$, suggesting that NsALD could induce the apoptosis of host cells through the intrinsic/Bcl-2-regulated/mitochondrial pathway.

In this study, NsALD was verified to be a secreted protein of $N$. seriolae by shotgun mass spectrometry and target the mitochondria in fish cells. The overexpression of the NsALD protein caused obvious apoptotic characteristics, such as the increase of the activity of caspase-3, the decrease of the $\Delta \Psi_{\mathrm{m}}$ values in FHM cells, and the significantly induced expressions of pro-apoptotic genes, indicating that NsALD can induce cell apoptosis. This study has laid the foundation for further clarification of the function of NsALD and provided new insights into the understanding of the molecular pathogenicity of N. seriolae.

\section{DATA AVAILABILITY STATEMENT}

The original contributions presented in the study are included in the article/Supplementary Material, further inquiries can be directed to the corresponding author/s.

\section{ETHICS STATEMENT}

This research did not include live animals. All animal experimental procedures involved were carried out in accordance with the Regulations for Animal Experimentation of GuangDong Ocean University, and the animal facility was based on the National Institutes of Health guide for the care and use of the Laboratory (NIH Publications No. 8023).

\section{AUTHOR CONTRIBUTIONS}

LX and YLiu designed the experiments. GC, LX, ZT, YLu, and TW performed the experiments. GC, LX, and ZT contributed to analysis. GC and LX wrote the paper. YLiu polished the paper. All authors contributed to the article and approved the submitted version.

\section{FUNDING}

This work was supported by the National Key Research and Development Project (2020YFD0900201), the Natural Science Foundation of Guangdong Province (2021A1515011222), the Key Research and Development Projects in Guangdong Province (2020B0202010009), and the Shenzhen Dapeng New District special fund for industry development (KJYF202001-08).

\section{ACKNOWLEDGMENTS}

We are grateful to all the laboratory members for their constructive suggestions to improve the manuscript.

\section{SUPPLEMENTARY MATERIAL}

The Supplementary Material for this article can be found online at: https://www.frontiersin.org/articles/10.3389/fvets. 2021.801990/full\#supplementary-material 


\section{REFERENCES}

1. Valdez IE, Conroy DA. The study of a tuberculosis-like condition in neon tetras (hyphessobrycon innesi). II. Characteristics of the bacterium isolated. Microbiol Espanola. (1963) 16:249-53.

2. Ho PY, Byadgi O, Wang PC, Tsai MA, Liaw LL, Chen SC. Identification, molecular cloning of il-1 beta and its expression profile during Nocardia seriolae infection in largemouth bass, Micropterus salmoides. Int $J$ Mol Sci. (2016) 17:1670. doi: 10.3390/ijms171 01670

3. Xia L, Cai J, Wang B, Huang Y, Jian J, Lu Y. Draft genome sequence of Nocardia seriolae ZJ0503, a fish pathogen isolated from Trachinotus ovatus in China. Genome Announcements. (2015) 3:e01223-14. doi: 10.1128/genomeA.01223-14

4. Manrique WG, Da SCG, de Castro MP, Petrillo TR, Figueiredo MA, Belo MA, et al. Expression of cellular compo nents in granulomatous inflammatory response in piaractus mesopotamicus model. PLoS ONE. (2015) 10:e0121625. doi: 10.1371/journal.pone.0121625

5. Maekawa S, Yoshida T, Wang PC, Chen SC. Current knowledge of nocardiosis in teleost fish. J Fish Dis. (2018) 41:413-9. doi: 10.1111/jfd.12782

6. Shimahara Y, Nakamura A, Nomoto R, Itami T, Chen SC, Yoshida T. Genetic and phenotypic comparison of Nocardia seriolae isolated from fish in Japan. J Fish Dis. (2008) 31:481-8. doi: 10.1111/j.1365-2761.2008.0 0920.x

7. Wang W, Chen J, Liao B, Xia L, Hou S, Wang Z, et al. Identification and functional characterization of Histone-like DNA-binding protein in Nocardia seriolae (NsHLP) involved in cell apoptosis. J Fish Dis. (2019) 42:657-66. doi: $10.1111 /$ jfd.12962

8. Rio-Odriguez RE, Ramirez-Paredes JG, Soto-Rodriguez SA, Shapira Y, Haydon DJ. First evidence of fish nocardiosis in mexico caused by Nocardia seriolae in farmed red drum (Sciaenops ocellatus, Linnaeus). J Fish Dis. (2021) 44:1117-30. doi: 10.1111/jfd.13373

9. Barry DP, Beaman BL. Nocardia asteroides strain GUH-2 induces proteasome inhibition and apoptotic death of cultured cells. Res Microbiol. (2007) 158:8696. doi: 10.1016/j.resmic.2006.11.001

10. Camp DM, Loeffler DA, Razoky BA, Tam S, Beaman BL, LeWitt PA. Nocardia asteroides culture filtrates cause dopamine depletion and cytotoxicity in PC12 cells. Neurochem Res. (2003) 28:1359-67. doi: 10.1023/A:10249444 31725

11. Beaman BL, Beaman L. Nocardia species: host-parasite relationships. Clin Microbiol Rev. (1994) 7:213-64. doi: 10.1128/cmr.7.2.213

12. Loeffler DA, Camp DM, Qu S, Beaman BL, LeWitt PA. Characterization of dopamine-depleting activity of Nocardia asteroides strain GUH2 culture filtrate on PC12 cells. Microb Pathog. (2004) 37:73-85. doi: 10.1016/j.micpath.2004.05.001

13. Hou S, Chen G, Wang W, Xia L, Wang Z, Lu Y. Identification of a cellwall peptidase (NlpC/P60) from Nocardia seriolae which induces apoptosis in fathead minnow cells. J Fish Dis. (2020) 43:571-81. doi: 10.1111/jfd. 13154

14. Hou S, Wang W, Chen G, Xia L, Wang Z, Lu Y. Identification of a secreted superoxide dismutase (SOD) from Nocardia seriolae which induces apoptosis in fathead minnow (FHM) cells. J Fish Dis. (2021) 44:63-72. doi: $10.1111 /$ jfd. 13268

15. Xia L, Liang H, Xu L, Chen J, Bekaert M, Zhang H, et al. Subcellular localization and function study of a secreted phospholipase C from Nocardia seriolae. Fems Microbiol Lett. (2017) 364:fnx143. doi: 10.1093/femsle/ fnx143

16. Huang YC, Jian JC, Wu ZH, Lu YS, Yu TJ. Isolation and identification of the pathogen causing sarcoidosis of Trachinotus ovatus. J Guangdong Ocean Univ. (2008) 4:49-53. doi: 10.3969/j.issn.1673-9159.2008.04.011

17. Gravell M, Malsberger RG. A permanent cell line from the fathead minnow (Pimephales promelas). Ann N Y Acad Sci. (1965) 126:555-65. doi: 10.1111/j.1749-6632.1965.tb14302.x

18. Feng Z, Cáceres NE, Sarath G, Barletta RG. Mycobacterium smegmatis Lalanine dehydrogenase (Ald) is required for proficient utilization of alanine as a sole nitrogen source and sustained anaerobic growth. J Bacteriol. (2002) 184:5001-10. doi: 10.1128/jb.184.18.5001-5010.2002
19. Dave UC, Kadeppagari RK. Alanine dehydrogenase and its applications-A review. Crit Rev Biotechnol. (2019) 39:648-64. doi: 10.1080/07388551.2019.1594153

20. Wiame JM, Pierard A. Occurrence of an $\mathrm{L}(+)$-alanine-dehydrogenase in Bacillus subtilis. Nature. (1955) 176:1073-5. doi: 10.1038/1761073b0

21. Hutter B, Singh M. Properties of the $40 \mathrm{kDa}$ antigen of Mycobacterium tuberculosis, a functional L-alanine dehydrogenase. Biochem J. (1999) 343:669-72. doi: 10.1042/0264-6021:34 30669

22. Chan K, Knaak T, Satkamp L, Humbert O, Falkow S, Ramakrishnan L. Complex pattern of Mycobacterium marinum gene expression during long-term granulomatous infection. Proc Nat Acad Sci. (2002) 99:3920-5. doi: 10.1073/pnas.002024599

23. Andersen $\mathrm{AB}$, Andersen $\mathrm{P}$, Ljungqvist L. Structure and function of a 40,000-molecular-weight protein antigen of Mycobacterium tuberculosis. Infect Immun. (1992) 60:2317-23. doi: 10.1128/IAI.60.6.2317-232 3.1992

24. Kitada S, Uchiyama T, Funatsu T, Kitada Y, Ogishima T, Ito A, et al. protein from a parasitic microorganism, Rickettsia prowazekii, can cleave the signal sequences of proteins targeting mitochondria. J Bacteriol. (2007) 189:844-50. doi: 10.1128/JB.01261-06

25. Kenny B, Jepson M. Targeting of an enteropathogenic Escherichia coli (EPEC) effector protein to host mitochondria. Cell Microbiol. (2000) 2:579-90. doi: 10.1046/j.1462-5822.2000.0 0082.x

26. Nougayrède JP, Donnenberg MS. Enteropathogenic Escherichia coli EspF is targeted to mitochondria and is required to initiate the mitochondrial death pathway. Cell Microbiol. (2004) 6:1097-111. doi: 10.1111/j.1462-5822.2004.0 0421.x

27. Papatheodorou P, Domańska G, Öxle M, Mathieu J, Selchow O, Kenny B, et al. The enteropathogenic Escherichia coli (EPEC) Map effector is imported into the mitochondrial matrix by the TOM/Hsp70 system and alters organelle morphology. Cell Microbiol. (2006) 8:677-89. doi: 10.1111/j.1462-5822.2005.00660.x

28. Palframan SL, Kwok T, Gabriel K. Vacuolating cytotoxin A (VacA), a key toxin for Helicobacter pylori pathogenesis. Front Cell Infect Microbiol. (2012) 2:92. doi: $10.3389 /$ fcimb. 2012.00092

29. Galluzzi L, Kepp O, Kroemer G. Mitochondria: master regulators of danger signalling. Nat Rev Mol Cell Biol. (2012) 13:780-8. doi: 10.1038/nrm3479

30. Lobet E, Letesson JJ, Arnould T. Mitochondria: a target for bacteria. Biochem Pharmacol. (2015) 94:173-85. doi: 10.1016/j.bcp.2015.02.007

31. Kozjak-Pavlovic V, Ross K, Rudel T. Import of bacterial pathogenicity factors into mitochondria. Curr Opin Microbiol. (2008) 11:9-14. doi: 10.1016/j.mib.2007.12.004

32. Layton AN, Brown PJ, Galyov EE. The Salmonella translocated effector SopA is targeted to the mitochondria of infected cells. J Bacteriol. (2005) 187:3565-71. doi: 10.1128/JB.187.10.3565-357 1.2005

33. Galmiche A, Rassow J. Targeting of Helicobacter pylori VacA to mitochondria. Gut Microbes. (2010) 1:392-5. doi: 10.4161/gmic.1.6.13894

34. Galmiche A, Rassow J, Doye A, Cagnol S, Chambard JC, Contamin S, et al. The N-terminal $34 \mathrm{kDa}$ fragment of Helicobacter pylori vacuolating cytotoxin targets mitochondria and induces cytochrome c release. EMBO J. (2000) 19:6361-70. doi: 10.1093/emboj/19.23.6361

35. Chen J, Xia L, Wang W, Wang Z, Hou S, Xie C, et al. Identification of a mitochondrial-targeting secretory protein from Nocardia seriolae which induces apoptosis in fathead minnow cells. J Fish Dis. (2019) 42:1493-507. doi: $10.1111 /$ jfd. 13062

36. Wang W, Hou S, Chen G, Xia L, Chen J, Wang Z, et al. Characterization and function study of a glutamyl endopeptidase homolog from Nocardia seriolae. J Fish Dis. (2021) 44:813-21. doi: 10.1111/jfd. 13311

37. Mehlen P, Bredesen DE. The dependence receptor hypothesis. Apoptosis Int J Programmed Cell Death. (2004) 9:37-49. doi: 10.1023/B:APPT.0000012120.66221.b2

38. Reed JC. Cytochrome c: Can't live with it - can't live without it. Cell. (1997) 91:559-62. doi: 10.1016/S0092-8674(00)80442-0 
39. Tischner D, Woess C, Ottina E, Villunger A. Bcl-2-regulated cell death signalling in the prevention of autoimmunity. Cell Death Dis. (2010) 1:e48. doi: $10.1038 /$ cddis. 2010.27

Conflict of Interest: The authors declare that the research was conducted in the absence of any commercial or financial relationships that could be construed as a potential conflict of interest.

Publisher's Note: All claims expressed in this article are solely those of the authors and do not necessarily represent those of their affiliated organizations, or those of the publisher, the editors and the reviewers.
Any product that may be evaluated in this article, or claim that may be made by its manufacturer, is not guaranteed or endorsed by the publisher.

Copyright $\odot 2022$ Chen, Tan, Liu, Weng, Xia and Lu. This is an open-access article distributed under the terms of the Creative Commons Attribution License (CC BY). The use, distribution or reproduction in other forums is permitted, provided the original author(s) and the copyright owner(s) are credited and that the original publication in this journal is cited, in accordance with accepted academic practice. No use, distribution or reproduction is permitted which does not comply with these terms. 